Revue des patrimoines

33 | 2017

Patrimoine culturel immatériel et institutions patrimoniales

\title{
Patrimoine vivant et contributions citoyennes. Penser le patrimoine « devant » l'Anthropocène
}

Living heritage and citizens' initiatives. Thinking about the heritage in relation to the Anthropocene

Jean-Louis Tornatore

(2) OpenEdition

Journals

Édition électronique

URL : http://journals.openedition.org/insitu/15606

DOI : 10.4000/insitu. 15606

ISSN : 1630-7305

Éditeur

Ministère de la culture

Référence électronique

Jean-Louis Tornatore, «Patrimoine vivant et contributions citoyennes. Penser le patrimoine « devant » l'Anthropocène », In Situ [En ligne], 33 | 2017, mis en ligne le 20 novembre 2017, consulté le 03 mai 2019. URL : http://journals.openedition.org/insitu/15606 ; DOI : 10.4000/insitu.15606

Ce document a été généré automatiquement le 3 mai 2019.

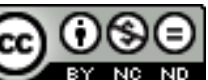

In Situ Revues des patrimoines est mis à disposition selon les termes de la licence Creative Commons Attribution - Pas d'Utilisation Commerciale - Pas de Modification 4.0 International. 


\title{
Patrimoine vivant et contributions citoyennes. Penser le patrimoine « devant » l'Anthropocène
}

\author{
Living heritage and citizens' initiatives. Thinking about the heritage in relation \\ to the Anthropocene
}

Jean-Louis Tornatore

"Alors que la commercialisation et les incitations
du marché exercent aujourd'hui une domination
incontestable, on peut néanmoins déceler parmi
les producteurs culturels une foule de courants
sous-jacents dissidents et de nombreux signes de
mécontentement susceptibles de faire un terreau
de choix pour l'expression critique et l'agitation
politique en vue de la production d'un nouveau
genre de communs. »1
"Quelles pensées pensent les pensées importe. »

1 Les études patrimoniales (ou heritage studies si l'on cède au point de vue anglo-saxon) sont aujourd'hui un véritable domaine de recherche avec ses courants, ses variations disciplinaires, ses singularités nationales ou culturelles, dont l'expansion remarquable en une vingtaine d'années se lit dans «une frénésie de publications, de colloques, d'événements et d'expositions " comme jamais le monde académique n'en aurait connu, si l'on en croit Ellen Hertz et Susan Chappaz ${ }^{3}$. Revers de la médaille (ou simple marque du destin académique), il a désormais toutes les caractéristiques d'un champ bourdieusien en pleine crise de croissance : luttes de prééminence, développements parallèles et emprunts souterrains à la faveur d'une faible perméabilité disciplinaire, ressassement ou réitération quasi rituelle de quelques motifs, notions, idées, concepts jugés remarquables... Parmi ceux-ci, deux thèmes ont aujourd'hui le vent en poupe. Tout d'abord, le thème du débordement de l'activité patrimoniale de son cadre institutionnel: l'idée selon laquelle cette activité est non seulement mise en œuvre à différents niveaux 
de la puissance publique (État, régions, communes...) mais procède également d'initiatives venues de ce qu'on appelle la "société civile» (individus, collectifs, associations) est désormais devenue le passage obligé de toute réflexion sur le patrimoine, le plus souvent doctement asséné et clamé comme trouvaille sensationnelle4. Eurêka ! Le nationalisme jacobin est définitivement mort et le Patrimoine, à l'instar de sa grande sœur, la Culture, est entré en démocratie! Du moins fait florès l'idée d'une "démocratie patrimoniale ", expression qui rappelle un débat qui, un temps, a agité le ministère de la Culture, à ceci près qu'elle désigne une activité qui ne concerne désormais que pour une part les institutions patrimoniales, puisqu'elles ont perdu leur monopole de l'action sur le passé. Mais qu'en est-il au juste? Expression commodément fourre-tout? Injonction auto-réalisatrice? Un deuxième thème alors se profile, celui d'un " patrimoine vivant ", qui serait un indice du débordement; du moins, par son allure d'oxymore, l'expression, issue de la catégorie de PCI, contiendrait une remise en question de la conception monumentale-nationale du patrimoine et mettrait en porte-à-faux les institutions patrimoniales françaises. Mais est-ce si sûr? Ces dernières sont-elles totalement démunies et/ou réellement menacées? La charge est-elle si subversive qu'on le dit? Ou alors, quand et comment l'est-elle? Je voudrais revenir sur ces thèmes à ma manière, c'est-à-dire en posant que c'est là affaire de posture : à quelles conditions peuton soutenir l'idée de démocratie patrimoniale comme celle de patrimoine vivant? Telle est finalement la question à laquelle je tente de répondre ${ }^{5}$.

\section{Débordements : lieux de mémoire et patrimoine ethnologique}

On oublie un peu vite que le phénomène (débordement-démocratie) a été pointé dès la fin des années 1970 sur le ton de la déploration, voire de la dénonciation, par les historiens de la mémoire nationale menés tambour battant par Pierre Nora. Orchestrés de main de maitre, ses Lieux de mémoire sont porteurs d'une inquiétude sourde quant à l'affaiblissement de cette mémoire nationale sous les coups des «mémoires particulières » demandant reconnaissance ${ }^{6}$. On pourrait objecter un peu rapidement que la mémoire n'est pas le patrimoine; or, une lecture un tant soit peu attentive des textes de scansion des Lieux par son architecte montrera que pour celui-ci le patrimoine n'est autre que l'objectivation, au sens propre du mot, la concrétisation si l'on veut, de la mémoire historique. On comprend dès lors qu'il fasse le constat de son émiettement et que « la tyrannie des mémoires » à laquelle serait confrontée la France des années 1970 ne soit que l'autre face de la prolifération des patrimoines. Jusqu'au tournant du siècle, c'est une appréhension négative du phénomène qui domine, tant est prégnante la voix des institutions patrimoniales d'État, qui, fortes de leur structure scientifiqueadministrative, fonctionnent à plein comme un dispositif de référence - tout est mesuré à l'aune de la patrimonialisation d'État - et il n'y a guère qu'un Jean-Michel Leniaud pour faire entendre, dès les années $1990^{8}$, un autre son de cloche, celui d'une attention à l'apparition de nouveaux médiateurs ("associatifs, élus, provinciaux ») des "nouveaux patrimoines » - « au détriment des habituels professionnels, les fonctionnaires spécialisés ${ }^{9} »-$, celui d'un plaidoyer pour le droit à la mémoire et la reconnaissance de « la diversité des identités et des histoires liées aux "lieux"10». Or, remarque-t-il, c'est précisément l'État qui aurait introduit un loup démocratique dans la bergerie nationale patrimoniale : «Il a fallu, écrit-il dans un article paru en 1993 à l'occasion de la sortie des derniers 
volumes de Lieux de mémoire, le "printemps" de l'année du Patrimoine en 1980, véritable signe précurseur de l'affirmation d'un droit à l'identité mémorielle, pour qu'apparaissent au grand jour des masses de patrimoines jusqu'alors clandestines $»^{11}$. Point de vue somme toute remarquable (et remarqué1 ${ }^{12}$ ) pour cet historien de l'art, archiviste de formation, et qui fut inspecteur des Monuments historiques dans les années 1980. On ne s'étonnera pas alors qu'il ait porté un regard ouvert et intéressé sur l'autre initiative remarquable de l'État patrimonial, la création, cette même année mémorable, de la Mission du patrimoine ethnologique (MPE) : invitant les ethnologues à fourrer leur nez dans les monuments historiques, à mettre en lumière leurs usages populaires ${ }^{13}$, y voyant le moyen de « mettre en évidence, à côté de celle des acteurs institutionnels, l'action des populations dans la définition du patrimoine ${ }^{14} »$.

3 Je serais tenté de voir dans le «moment du patrimoine ethnologique » l'envers positif du «moment des lieux de mémoire "- hypothèse confortée par le fait que ces deux expériences concomitantes se sont superbement ignorées: alors que la seconde, mobilisant majoritairement des historiens, s'ingéniait à cartographier les «buttes-témoin ${ }^{15}$ » de la mémoire-histoire, de manière à (re)composer le roman national, la première, aux mains des ethnologues, mais attirant très vite sociologues, géographes, urbanistes, architectes, etc., offrait une photographie empathique, captivante et variée d'une société saisie par la «fièvre patrimoniale ${ }^{16}$ ». Du moins le moment avait ceci d'excitant qu'il offrait la possibilité aux ethnologues et agents de la Mission du patrimoine ethnologique comme aux chercheurs engagés dans les programmes élaborés et pilotés par le Conseil du patrimoine ethnologique d'assister in vivo ou "grandeur nature » aux manifestations de cette fièvre, voire d'y participer. L'expérience n'a sans doute pas été la même selon qu'on la vivait depuis « la Centrale » - en fait la MPE proprement dite - ou ses programmes, ou bien depuis une position plus proche du terrain - un terrain complexe combinant le culturel, le politique et l'administratif -, telle celle qu'occupaient les «conseillers à l'ethnologie » affectés dans les directions régionales des Affaires culturelles, et la différence a pu se faire sentir dans la manière de problématiser le phénomène. Comment penser le patrimoine à l'heure de son inflation, de la prolifération de ses objets? « Nous autres ethnologues, "sommes embarqués" »" déclarait en 1993 Daniel Fabre, alors viceprésident du Conseil du patrimoine ethnologique, en conclusion d'un colloque qui se tenait à Tours ${ }^{18}$, tant la discipline lui paraissait en mesure de donner une intelligibilité à une situation inédite de chamboulement «du rapport entre patrimoine, identité et nation » - mots d'alors - dans l'espace européen. Il est notable que, faisant terrain de ses positions dans l'institution patrimoniale, depuis sa participation au groupe de travail qui, sous la présidence de Redjem Benzaïd, jeta les bases de la politique du patrimoine ethnologique jusqu'à sa participation au dernier Conseil du patrimoine ethnologique et à la création d'un laboratoire de recherche rattaché au ministère, il a élaboré une approche qui fait du tournant patrimonial une manifestation majeure de "l'institution de la culture », processus qui voit la culture objectivée par ses propres représentants ${ }^{19}$, et du patrimoine ethnologique un événement majeur dans l'histoire de ce tournant. Cependant, son souci d'une mise en perspective ethno-historique l'orientera vers une conception lettrée, esthétisante et irénique - non dénuée d'accents symbolistes - du phénomène patrimonial contemporain et de la contribution de l'ethnologie à sa compréhension. De ce point de vue, sa communication aux Entretiens du patrimoine de novembre 1994, placés sous la présidence de Pierre Nora, dans laquelle il commente, justifie et problématise la relation instituée entre patrimoine et ethnologie, est un monument de diplomatie, tant il 
réussit à montrer que la jeune ethnologie de France s'est construite sur des questions proprement patrimoniales (la transmission et la continuité sociale, le populaire, les derniers souffles d'une société - partant une sensibilité toute ethnologique à l'urgence), sa capacité à fonder de l'action patrimoniale (sensibilité aux formes artistiques et expressives, restitution de la dimension immatérielle ou symbolique de tout monument, engagement dans des processus d'inventaire et de conservation naturaliste), tout autant que sa capacité à introduire des ruptures dans l'agir patrimonial officiel, à « faire bouger la notion » (par introduction de l'immatériel, du présent, de l'objet-système qui fait sens, de la relativité ethnographique) et, au final, à contribuer à «l'éveil d'une conscience critique du patrimoine ${ }^{20} »$. En somme, le patrimoine attendait l'ethnologie. Du point de vue du terrain, les réponses furent moins portées par un esprit de discipline qu'intéressées à comprendre l'enjeu du débordement institutionnel et à prendre la mesure des forums hybrides qui se déployaient sous les yeux des ethnologues « embedded »: des objets, des acteurs, leurs ressources, les sens qui s'élaboraient ou se coconstruisaient dans l'action...; bref, à donner son épaisseur sociale à la nouvelle et intense activité patrimoniale. Michel Rautenberg problématisait l'action de l'ethnologue en région (et en DRAC) en «intervention ethnologique ${ }^{21}$ » et proposait une analyse de la double tension entre le patrimoine régalien et les " patrimoines sociaux » et entre les mémoires collectées et la mémoire collective en termes de continuités et de ruptures ${ }^{22}$. Noël Barbe explorait en quoi consistait désormais une "action patrimoniale ", soit une pratique sociale de narrativité du passé émergeant de la confrontation entre experts et profanes, et proposait une des premières approches pragmatiques ${ }^{23}$. Quand, de mon côté, après avoir suggéré de mobiliser la notion d'objet-frontière pour penser le patrimoineaujourd'hui ${ }^{24}$ et m'appuyant sur le couple notionnel politisation/cristallisation élaboré par le sociologue Jean-Yves Trépos, posant que l'expertise n'est pas réservée aux seuls experts $^{25}$, j'explorais les formes d'engagement dans/de l'activité patrimoniale ${ }^{26}$ et m'attachais à comprendre la patrimonialisation de la Lorraine industrielle, soit une intrication d'actions top-down et bottom-up composant ce que j'ai appelé un « espace de la mémoire $^{27} »$.

\section{Des espaces publics du patrimoine? Entre démocratisation et marchandisation}

Ce bref retour n'a d'autre visée que de souligner la richesse pratico-théorique de ces années : en ce sens, «le moment du patrimoine ethnologique ${ }^{28}$ » peut être considéré à la fois comme l'expression emblématique de "l'âge du patrimoine", voire son point d'orgue, et comme un poste d'observation-participation sans égal du phénomène patrimonial. On pourrait cependant aller plus loin en considérant que le patrimoine ethnologique s'est trouvé au cœur d'un âge du patrimoine profondément ambivalent ambivalence dont «l'ethnologie patrimoniale » n'a sans doute pas suffisamment pris la pleine mesure. Abruptement, je dirais aujourd'hui qu'une vision, mettons, «enchantée " le dispute à une version sans doute plus réaliste. La première considère que, dès les années 1970, "l'expérience du passé " se confond avec celle de la culture et devient l'affaire de tous, une expérience grâce à laquelle tout un chacun, constitué en collectif i. e. se thématisant en collectif - peut faire valoir ce qu'il est au moyen de ce qu'il a et construit ainsi son identité dans le temps. On reconnaît la thèse suggestive de Daniel Fabre selon laquelle l'âge du patrimoine se singularise dans le glissement de l'avoir à 
l'être: «Le patrimoine, c'est nous! $»^{29}$. L'âge du patrimoine, c'est donc l'âge de sa démocratisation : prenant le pas sur le monument - mais sans le renier -, le patrimoine devient culturel puis ethnologique ; le périmètre de ce qui doit être distingué en même temps que la pratique même de distinction sont étendus au peuple et à la société. Vue sous cet angle, cette extension ne procède pas de la rupture : on remarquera la cooccurrence du développement industriel et des politiques patrimoniales et, au moment du constat de l'emballement patrimonial, l'idée est avancée que la forme d'appropriation spécifique (collective et par conséquent imposant des limitations), constitutive du patrimoine, s'oppose à celle liée à la consommation destructrice. Thèse défendue, par exemple, par Roland Schaer : « Le rapport au patrimoine s'apparente au rapport à l'œuvre d'art: un désir suspendu arrêté au seuil de la dévoration, qui permet de transmettre, inentamée, une richesse tenue pour inépuisable $\aleph^{30}$. Il s'ensuit que dans la perspective de "dilatation du patrimoine [...], cette appropriation s'oppose à l'appropriation technicienne du monde, qui le prend pour pur objet à transformer $»^{31}$. Dès 1980, Marc Guillaume avait cependant envisagé de manière plus nuancée la relation entre capitalisme et patrimoine, perspective qui ouvre sur la version réaliste de la dilatation. Selon lui, grâce à la valeur cardinale de pérennité, la conservation viserait à compenser la destruction qui accompagne le développement industriel : elle s'afficherait ainsi comme "contre-dépendante de la production-consommation marquée par l'éphémère et l'obsolescence ", de manière à " rendre acceptable l'accélération de l'usure du quotidien » 32. Et de voir dans la récente archéologie industrielle et la sensibilité patrimoniale naissante pour les objets de culture industrielle - Concorde, par exemple, entré au musée comme chef-d'œuvre de technologie ${ }^{33}$ - une forme de célébration de la «société de consommation et de la croissance triomphante ${ }^{34}$ ». En d'autres termes, l'âge du patrimoine verrait une autre évolution, cette fois-ci en forme de rupture, au terme de laquelle le patrimoine pourrait bien ne plus être ce qu'il a été sous le signe du monument ou de l'objet pétri de valeur d'art et/ou d'histoire. En effet, sous couvert de cette diffusion-émiettement de la cause patrimoniale, s'est développée une instrumentation à la fois politique et économique du patrimoine. Si la première a été assez bien repérée ${ }^{35}$, la seconde l'a été beaucoup moins; en tout cas, le patrimoine est devenu un argument significatif de "développement", maître-mot performatif du local au national, dont l'usage se répand précisément au même moment.

5 Ainsi, s'il est vrai que dans le temps du patrimoine ethnologique prend forme un « espace public » du patrimoine $\mathrm{e}^{36}$, il y a lieu dès lors de considérer le fait que son extraordinaire, sinon monstrueux, déploiement aujourd'hui dans les mondes académiques, associatifs, administratifs, politiques, économiques, etc., ne connaît guère de limites et que, somme toute, l'argument patrimonial est développé dans des registres, voire dans des régimes, très différents. Pour donner un aperçu de la complexité de cet espace public pluriel et son débordement sur les sphères marchandes, partons d'un commentaire sous la plume de quatre géographes qui ouvre un ouvrage collectif récent qui se donne pour objectif d'explorer la part du patrimoine dans le développement rural :

Le patrimoine constitue aujourd'hui un enjeu majeur de société. Il est une transmission, une forme d'héritage qui peut être saisie collectivement ou individuellement. Objet composite et transversal, mobilisé par les acteurs, il conduit souvent à des formes complexes de développement territorial. S'appuyant sous sur des ressources matérielles ou immatérielles, elles-mêmes produites de l'histoire, il s'avère être un levier parfois décisif dans la mise en place des politiques de développement local. Ces dernières, à l'interface entre patrimoine, ressources et 
territoires, se basent sur des interactions qui dépendent beaucoup de la capacité des acteurs à révéler et valoriser leurs ressources territoriales ${ }^{37}$.

La géographie économique, particulièrement, a été "efficace " à saisir l'intrusion de ressources non marchandes dans le développement des territoires. Les ressources culturelles, entendre patrimoniales, s'insèrent dans une conception de la territorialité pensée en termes de trajectoire, de compétition et de compétitivité, et pour laquelle joue à plein la promotion des localités et des singularités ${ }^{38}$. Dans un jargon triste de management territorial, le patrimoine comme ressource devient une "offre de produit " (tels des produits «leader » bénéficiant d'un label AOC) ou une " offre de site » pouvant constituer, pour un territoire, un " panier de biens »; l'association entre territoire, biens typiques et demande appropriée se renforçant mutuellement sous la forme d'une « rente de qualité territoriale ${ }^{39} »$. Cette instrumentation du patrimoine comme levier décisif du développement local et donc concourant à formater en ressources des éléments culturels matériels ou immatériels vient à mon sens fortement nuancer la proposition selon laquelle l'âge du patrimoine entérinerait une identification de collectifs à un/leur patrimoine. «Le patrimoine, c'est nous », certes, mais le patrimoine est aussi et toujours ces travaux l'attestent - un bien dont « on » (Qui ? Là est la question!) tire avantage, bénéfice, plus-value. Bien que les auteurs des études ci-dessus mentionnées avancent que la valorisation économique n'est ni systématique ni obligatoire, qu'elle n'est qu'une étape optionnelle dans la "démarche d'exposition du patrimoine ${ }^{40}$ ", on peut cependant se demander si l'ensemble du processus de transformation patrimoniale d'éléments ou de biens culturels ne prépare pas, sinon ne vise pas, l'usage marchand. Le parallèle entre les paniers de biens et les services écosystémiques incite à cette généralisation : les seconds instaurent une relation "entre un écosystème bénéfique et un individu (ou groupe d'individus) bénéficiaire ${ }^{41}$ » et en ce sens, une "nature à vendre»; la patrimonialité territoriale repose de même sur le principe d'une " culture à vendre ». La version réaliste de l'emballement patrimonial fait un pas supplémentaire par rapport à la thèse de Marc Guillaume $^{42}$ : on voit combien le patrimoine a su faire bon ménage avec le capitalisme version «nouvel esprit »!

7 Significativement, l'approche juridique vient fournir un élément d'analyse de cette tendance. Commentant la position du patrimoine "entre l'avoir et l'être ", Françoise Fortunet repère une mutation radicale du sens du concept: d'une identification à l'être (le patrimoine est originairement lié à la personne, au sujet), le patrimoine se trouve « rattaché à l'objet ou à la cause qui en justifie l'emploi »:

Parler de patrimonialité revient à pouvoir donner une valeur pécuniaire à un bien et ainsi à le faire entrer dans un cercle d'échanges auxquels des biens de même espèce peuvent être affectés, autrement dit à le faire entrer sur un marché où sa valeur serait une marque d'identité substantielle ${ }^{43}$.

Est-ce à dire que la politique du patrimoine porte en germe la marchandisation de la culture? Les travaux actuels de Luc Boltanski et Arnaud Esquerre sont bienvenus qui montrent que la singularisation et l'appropriation de choses et de produits qui ont du prix, des objets patrimoniaux aux produits de luxe, participent d'une économie de l'enrichissement, consécutive à la désindustrialisation ${ }^{44}$. Dans cette perspective, le patrimoine est ce processus par lequel est légitimée « la forme collection », laquelle a pris une ampleur inédite au cours des dernières années, mais qui est contrainte par ce que les auteurs appellent la forme "actif»: «les choses sont pertinentes en tant qu'elles constituent un capital, autrement dit en tant que compte tenu de leur prix actuel, elles peuvent être considérées comme une source possible d'enrichissement exprimable en 
monnaie et de revenus futurs $»^{45}$. Pour autant, le géographe marxiste David Harvey ${ }^{46}$ relève que la marchandisation de la culture n'en est pas moins source de contradictions qui tiennent à la spécificité des produits culturels par rapport aux marchandises ordinaires. Pour le dire vite, si la culture, l'histoire et le patrimoine sont des lieux de constitution de rentes de monopole, ce "constant objet du désir capitaliste ${ }^{47}$ ", ce sont aussi, en tant que mettant en exergue les valeurs d'authenticité, de singularité, d'originalité, d'unicité ou de créativité, des lieux éminemment conflictuels, sources d'appropriations ou d'oppositions alternatives. Et d'appeler «à tout faire " pour que ces rentes ne soient pas le produit exclusif de la bourgeoisie, et pour persuader les "producteurs culturels contemporains" de s'opposer à la marchandisation, à la domination du marché et au capitalisme ${ }^{48}$. Si son analyse des mécanismes de la marchandisation de la culture n'est guère éloignée de celle des géographes aménageurs, elle se différencie par une position radicalement critique et d'engagement pour l'exploration, à partir du terrain patrimonial et culturel, «d'un autre type de relations sociales et écologiques ${ }^{49} »$. C'est ce type de perspective que je voudrais maintenant explorer.

\section{Le patrimoine comme expérience : pour un tournant pragmatiste}

9 L'environnement fait aujourd'hui l'objet d'une nouvelle histoire et d'une nouvelle géographie qui se disent respectivement «environnementales", et non pas «de l'environnement ${ }^{50}$ ", comme manière d'historiciser la relation à l'objet et d'engager une dimension de réflexivité; de même, il nous faut dépasser la simple perspective d'une ethnologie ou anthropologie du patrimoine et réfléchir à la possibilité d'une « anthropologie patrimoniale ». Si l'expression n'est pas dénuée d'ambiguïté, elle se situe dans le prolongement d'une réflexion initiée depuis l'expérience du patrimoine ethnologique. On a su certes en profiter pour donner naissance à une ethnologie du patrimoine $^{51}$, on n'a cependant pas suffisamment tiré tous les fils que cette expérience a tissés. Or, me semble-t-il, la conjoncture aujourd'hui, quelque chose comme l'état du monde et des humains dans le monde, offre une nouvelle opportunité pour le développement d'une approche non pas seulement réflexive du patrimoine mais pragmatiste, c'est-à-dire tout à la fois ouverte, située et impliquée.

10 «Ouverte " parce que le patrimoine n'est pas une réalité qui peut être segmentée a priori - du moins, il est opératoire de ne pas s'abandonner à un découpage a priori de la réalité patrimoniale. La complexité du patrimoine aujourd'hui, sa réalité plurielle, n'est pas à réduire ni un problème à résoudre; elle est à explorer en tant que telle; il faut en parcourir les multiples manifestations, en décliner toutes les composantes: en tant que relation au passé partageant cette fonction avec d'autres modalités du passé-présent (la mémoire, l'histoire...), mais aussi en tant que relation aux êtres et aux choses dans leur réalité présente et dans leur devenir - ce qui suppose de ne pas réduire le patrimoine au seul rapport au passé. S'agit-il pour autant d'engager une anthropologie globale du phénomène patrimonial ? Sur ce point, Daniel Fabre, soucieux d'historicité, se montrait prudent, du moins voulait-il rapporter le phénomène «relation au passé » à des temps successifs singularisés et réserver le mot patrimoine aux occurrences contemporaines de ce phénomène, y voir le symptôme d'un « ultime et singulier avatar des traces objectales du passés2 $»$. Certes, d'un point de vue scientifique, l'imprudent encourt le risque 
impardonnable de l'anachronisme. Pour autant, le déplacement que je propose suggère de se confronter au " problème du nominalisme » en intégrant dans l'analyse les usages des mots, ce qu'ils imposent, les mesures qu'ils accomplissent et le propre travail de réflexivité auquel se livrent les acteurs eux-mêmes : non pas donc une anthropologie globale du patrimoine, mais une anthropologie de l'activité, toute l'activité qui se dit / est dite patrimoniale aujourd'hui. En ce sens, mon approche ne veut pas être contrainte par des catégorisations préalables, celle de nos catégories communes inscrites dans le langage comme celles qui résulteraient des institutions d'administration du patrimoine ou qui seraient en particulier l'expression d'une "pré-occupation" scientifique liée au développement des savoirs contribuant à l'instruction de la valeur patrimoniale. Il importe de saisir tout ce dont le patrimoine peut être aujourd'hui le nom. En somme, je fais le pari d'une définition "englobante ", voire générique, apte à saisir les proximités et les conjonctions dans les expériences patrimoniales, que celles-ci visent la conservation, la célébration ou les usages non seulement (des traces) du passé dans le présent mais, audelà, de ce qui est défini par des personnes, des collectifs, des acteurs... comme ayant du prix et comme étant ce qu'il leur importe de sauvegarder.

11 "Située », parce que le patrimoine se concrétise dans des attachements ou des liaisons dans le présent des collectifs et se réalise dans la " pédagogie » de ses objets (i.e. ce qu'ils font faire) ; "située ", également en raison de la connaissance produite, à l'opposé de celle commise sous l'injonction d'une objectivité neutre ${ }^{53}$. Le mot, on le voit, est pris au sens de « référé à des objets » - et, nécessairement, contextualisé en tant qu'il renvoie à des situations - mais il renvoie aussi aux actions constitutives de la situation, à ce qui émerge de la dynamique des interactions ou des circonstances, auxquelles n'échappe pas l'analyste.

12 "Impliquée ", « concernée », " engagée »... Les qualificatifs ne sont pas stabilisés pour dire l'abandon de la trop indiscutée et commode neutralité axiologique. Peut-on en rester à un exposé froid de la complexe réalité patrimoniale - comme de toute réalité sociale ou culturelle? Dès le début des années 1990, la Mission du patrimoine ethnologique a été le lieu d'une réflexion, certes peu publicisée, sur l'implication du chercheur - en l'occurrence le conseiller à l'ethnologie ${ }^{54}$ - dans des processus partagés de connaissance et de valorisation d'éléments culturels, réflexion qui s'est traduite dans un tournant réflexif ${ }^{55}$, préfigurant dans une certaine mesure le « tournant participatif » que connaît le patrimoine aujourd'hui dans le cadre d'un «effet PCI». La raison académique n'a cependant pas manqué de minorer cet apport en concédant sagement le développement d'une ethnologie du patrimoine, laissant le débat se diluer dans l'opposition stérile entre la posture de l'ethnologue critique (celui qui dévoile et dénonce) et celle de l'ethnologue embarqué qui, en l'occurrence, rédige des fiches d'inventaire. Le débat est plus largement récurrent dans les sciences sociales, en témoignent des courants divers: sociologie publique, intervention sociologique, recherche collaborative..., voire l'idée d'une "science impliquée », visant le partage des savoirs et la mise en discussion de l'autorité scientifique, qui connaît aujourd'hui un certain renouveau ${ }^{56}$. Posons le problème ainsi : le pragmatisme est un art des conséquences, il reste alors à son producteur à assumer les conséquences des connaissances produites, ou plutôt à produire des connaissances pour leurs conséquences : celles-ci sont alors une participation à l'état du monde et à la fabrication de sa réalité. S'agissant du patrimoine, on ne peut en rester à la figure de l'ethnologue en performeur patrimonial, il faut se demander s'il est possible de penser des manières de "faire patrimoine » - i.e. de penser une opérativité du patrimoine - en 
dénouant le lien au capitalisme, soit à l'économie de l'enrichissement et à la dévastation écologique du monde.

Cette approche conduit à revenir sur un certain nombre d'idées reçues. Tout d'abord, le patrimoine ne dit pas tout du passé et n'est qu'une modalité parmi d'autres ayant pour fonction d'assurer la présence du passé dans le présent, mais dit autre chose que le passé. L'histoire de l'Occident moderne est jalonnée de topiques ou de lieux dans lesquels s'est matérialisée la "raison patrimoniale » : le monument, le lieu ou le site, la culture, le vivant ${ }^{57}$... Il s'ensuit que la diversité des "êtres" que consacre cette raison, sous l'attribution d'une valeur, qu'ils soient indifféremment de pierre, de chair, de matière organique, d'esprit, d'idée ou de mots, ne peut être saisie via le jeu des distinctions binaires qui équipent la doxa institutionnelle: mobilier/immobilier, monumental/ culturel, matériel/immatériel, culturel/naturel... Cette valeur sera en conséquence génériquement définie comme une disposition à dire et à instaurer «ce à quoi nous tenon ${ }^{58}$ ", quel que soit l'usage qui en est fait et qui concourt à instaurer une relation de dépendance - « ce à quoi nous tenons et dont nous dépendons ${ }^{59}$ ». Enfin, cette perspective conduit à mettre l'accent sur l'actualité du patrimoine, au sens où il instaure moins une valeur de transmission qu'une valeur d'actualité ou d'actualisation: le patrimoine ne se transmet pas, il s'actualise, il ne se transmet qu'à la condition qu'il s'actualise. L'empirisme pragmatiste - «radical» selon William James ${ }^{60}$, «immédiat " pour John Dewey ${ }^{61}$ - permet de préciser le déplacement de focale : le patrimoine est tel qu'il est " expériencé », il est à la fois la chose et les relations (ou transactions) qu'elle signifie et organise; il est l'expérience de ces relations, d'où l'importance des prépositions qui constituent autant de "clés d'interprétation ${ }^{62} »:$ le patrimoine, ce n'est toujours qu'un patrimoine-de, un patrimoine-pour, un patrimoine-avec, un patrimoine-contre, etc. Poser le patrimoine comme expérience, ce n'est pas seulement rendre compte de l'expérience du passée ${ }^{3}$ ou de l'expérience de la culture ${ }^{64}$, c'est conjointement faire valoir l'efficace de l'attachement instauré par une relation dite patrimoniale et le situer dans un enchaînement d'expériences qui engagent les activités mentale et émotionnelle et qui se déploient dans des registres esthétiques, artistiques, éthiques, politiques ou marchands. C'est donc le considérer en son actualité, de telle manière à comprendre sa diffraction dans les espaces publics (il n'est pas ou plus monopolisé par les institutions étatiques ou internationales) et, en conséquence, interroger son opérativité et sa puissance d'agir, en un mot son « agentivité ».

\section{Du patrimoine vivant}

14 À saisir cette actualité, la singularité du patrimoine aujourd'hui réside indéniablement dans son ouverture sur le vivant, ouverture permise par le passage historique, déjà évoqué, de l'âge du monument à l'âge du patrimoine et problématisée en tant que glissement du paradigme de la conservation au paradigme de la sauvegarde ${ }^{65}$. À partir d'un intérêt pour la nature et ses saisies patrimoniale et écologiste, André Micoud a sans doute été un des premiers, sinon le premier, à remarquer cette mutation fondamentale et à proposer une sorte de tableau synthétique, très suggestif, permettant de voir «toutes les patrimonialisations d'un seul coup d'œil», en les envisageant du point de vue du vivant : « le vivant qui a eu lieu (dont on doit conserver les traces) », « le vivant en passe de disparaître (dont on doit sauvegarder les restes) » et « le vivant plein de potentialités (dont il faut gérer la diversité) $\gg^{66}$. Son approche a ceci de remarquable qu'elle est une première 
entreprise forte de problématisation du phénomène patrimonial contemporain, construite autour de trois gestes : un, penser ensemble nature et culture dans ou sous la catégorie de patrimoine; deux, montrer, à partir d'une posture herméneutique, que les patrimonialisations (les trois moments : conservation, sauvegarde, gestion) offrent « trois manières de concevoir le rapport à ce qui vit »; trois, voir dans l'engouement pour le patrimoine la marque d' 'u un changement du rapport du temps au vivant» (différent du temps de la modernité ${ }^{67}$. C'est particulièrement au sein du paradigme de la sauvegarde que se réaliserait la jonction entre des préoccupations relatives à la culture, inaugurées dans les années 1960 avec l'essor de la notion de patrimoine culturel visant à élargir et dépasser une conception strictement monumentale ou artistique de la relation au passé, et des préoccupations liées à la nature, elles-mêmes thématisées dans la notion de patrimoine naturel.

15 André Micoud revendique une posture herméneutique qui l'affranchit de la contrainte historiciste ; les moments qu'il distingue, écrit-il, sont moins chronologiques que logiques ${ }^{68}$. On peut toutefois reprendre la question à partir des institutions. L'articulation du naturel et du culturel dans le patrimoine n'est pas nouvelle: elle est contenue dans les notions de site et de paysage culturel qui sont l'objet de lois ou d'instruments internationaux du patrimoine (en particulier la convention Unesco de 1972). Le fait nouveau résiderait alors dans les évolutions conjointes de la saisie patrimoniale des deux ordres de «notre » réalité. Avec la catégorie et la politique du patrimoine ethnologique, relayées par celles du patrimoine culturel immatériel, c'est bien sûr l'acception anthropologique de la culture qui prévaut et « dépasse » l'acception classique orientée sur les biens et les œuvres qui irrigue la conception malrucienne du patrimoine culturel. Du côté de la nature, on peut considérer une semblable évolution lisible dans les débats vifs qui agitent la galaxie écologiste: entre l'idée d'une nature extérieure à l'homme, objectivée et sanctuarisée - repérable dans la tradition états-unienne des grands parcs nationaux ou dans la perspective de l'écologie profonde - et l'idée d'une nature anthropisée, telle que développée par les courants de l'écologie sociale, de l'environnementalisme social ou les mouvements pour la justice environnementale ${ }^{69}$.

Pour autant, on n'échappe pas à la possibilité de deux lectures contradictoires. La première minorerait le changement. Sans doute peut-on considérer que le patrimoine culturel immatériel assure un basculement de la topique de la culture à la topique du vivant. C'est précisément la convention Unesco de 2003 qui va populariser l'expression « patrimoine vivant » en assurant la promotion de pratiques expressives plutôt que celle d'objets, produits de la créativité humaine, en se présentant comme un pavé dans la mare de la patrimonialité occidentale. Pour autant, avant même d'avoir vu la convention en fonctionnement, il était possible de douter de son caractère « révolutionnaire » et de remarquer que la convention réintroduisait un fonctionnement « en régime d'objet » que précisément la catégorie de patrimoine ethnologique avait tenté de subvertir ${ }^{70}$. En effet, l'échec de la politisation du patrimoine ethnologique a tenu précisément en ce qu'il explorait un mode de (re)connaissance de la culture qui ne coïncidait pas avec la logique objectale de la politique patrimoniale centrée sur les monuments et les œuvres. S'il y a une différence à pointer entre patrimoine ethnologique et patrimoine culturel immatériel, qui va de pair d'ailleurs avec la minoration de l'expertise ethnologique ${ }^{71}$, c'est bien le fait que la seconde catégorie vise, s'agissant de la culture anthropologique, la singularisation d'élément culturels auxquels est attribuée une fonction d'emblème ou de représentation, autrement dit qu'elle réintroduit un mode de traitement en objet, 
l'appliquant à l'immatérialité culturelle. Les politiques du PCI ne manquent pas d'alimenter la «forme collection ${ }^{72}$ » constitutive de l'économie de l'enrichissement - ce n'est pas le cas du repas gastronomique des Français qui va le démentir, tant il révèle une forme de récupération au service d'une " aimable » compétition entre nations, instaurant une sorte de «mondial du patrimoine $»^{73}$. D'autre part, on peut toujours douter de la possibilité affirmée d'une réflexion en termes de "natureculture ", en un seul mot - ce à quoi s'échine vaillamment un Bruno Latour. Il y aurait une sorte de rétivité à cette transformation constitutive du mot patrimoine, lisible dans une séparation profonde entre patrimoine culturel et patrimoine naturel, entre leurs histoires et institutions respectives. À cette séparation s'ajoute le fait que, alors que le patrimoine apparaît historiquement comme un moyen privilégié de penser la culture dans son rapport au temps, d'assurer concrètement sa traversée du temps, il semble que la notion ne soit guère un thème fédérateur des positionnements environnementalistes ou écologistes. Du moins y a-t-il un hiatus entre des positionnements organisés autour de la monumentalisation, de la sanctuarisation de la nature ou de la notion de diversité biologique et des positionnements qui, associant l'environnemental au social, tentent de sortir de la tension entre vision anthropocentrée et vision biocentrée, avancée par l'écologie profonde, et se proposent de penser une «nature ordinaire», ou une "nature comme communauté", selon la proposition de Giovanna Di Chiro, c'est-à-dire de " réinventer la nature par l'action communautaire ${ }^{74}$ ».

Une autre lecture, que je propose, plus volontairement optimiste, consiste à voir dans l'émergence d'une topique patrimoniale du vivant une pensée radicalement différente du patrimoine, centrée sur deux motifs significatifs, étroitement liés. D'une part, un renversement de perspective : au motif de la représentation - aux sens conjoints de présenter de nouveau et rendre présent aux sens - du passé, lisible dans le monument comme dans le lieu - précisément dans le lieu de mémoire - ou encore dans l'indice culturel folklorisé thématisé comme survivance, vient s'ajouter celui de l'attention présente à porter pour le futur. Là est très certainement la nouveauté : si le temps, i.e. la fuite du temps et la manière de s'en accommoder, est le cadre usuel d'exercice du patrimoine, voilà que le regard (la raison de son inquiétude) change de direction : l'objet de la perte auquel il faut assurer la présence n'est pas seulement derrière nous, il est aussi devant nous, il est à-venir. La perte n'est pas à régler/solder, elle est à anticiper - sans doute parce qu'elle vient vers nous sous la figure désormais insistante de la catastrophe. D'autre part, une présence aux êtres et aux choses qui comptent : en ce sens, le sentiment d'étrangeté constitutif du monument ou de l'être patrimonial (l'objet "vitrinifié» du musée, par exemple) disparaît au profit de celui d'une proximité ou d'un proche. Les thèmes de la distance et de la neutralisation patrimoniales étaient déjà présents dans l'opposition entre mémoire-conservation symbolique et mémoire-conservation hétérologique sur laquelle Marc Guillaume appuyait sa critique de la politique du patrimoine $^{75}$. Or, précisément, la critique de la mise à distance, comme lieu de manipulation et de domination ou comme désenchantement et rationalisation du monde ${ }^{76}$, connaît un renouveau aujourd'hui, dans la perspective de repenser le lien aux êtres et aux choses, à laquelle ne peut être indifférente la réflexion sur l'opérativité du patrimoine aujourd'hui. 


\section{« Devant » l'anthropocène : retour à l'héritage ${ }^{77}$}

18 Le prolongement de cette lecture est la confrontation du thème d'une topique patrimoniale du vivant au " motif » de l'Anthropocène, en tant que condensé, à première vue, des grands enjeux du $\mathrm{xxI}^{\mathrm{e}}$ siècle. Cette désignation géologique de notre époque pointe le fait que l'humain est devenu un agent de la transformation radicale, immaîtrisée et sans doute irréversible de la Terre. En quelques années, elle s'est installée avec insistance dans les débats scientifiques et commence à prendre consistance dans l'opinion publique au regard des préoccupations croissantes quant à l'impact de l'activité humaine - plus souvent, d'ailleurs, envisagé à partir du changement climatique. Il est notable que, pour avoir été inventée au début des années 2000 par un chimiste de l'atmosphère (Paul Crutzen), historiens et philosophes de l'environnement, des sciences et des techniques, sociologues, anthropologues, géographes, tenants de l'écologie politique, sémioticiens, artistes n'ont pas attendu sa validation par la Commission internationale de stratigraphie (elle est toujours en examen à ce jour) pour s'emparer du mot et l'interroger dans ses implications historiques, politiques et ontologiques ${ }^{78}$.

19 Le concept d'Anthropocène cristallise en quelque sorte l'émergence d'un nouveau champ de recherche, les humanités environnementales, qui réunit toutes ces disciplines autour d'un commun intérêt à dépasser le partage entre une nature physique et une culture humaine et à penser notre nouvelle époque - les enjeux auxquels sont confrontés les humains - sous l'angle des "ontologies interconnectées ${ }^{79}$ ». Ce domaine en fort développement renouvelle un débat amorcé dans un premier temps, ou comme premier pas, par la notion de développement durable, laquelle voulait tenter l'articulation sinon une combinatoire de domaines distingués de la réalité, le social, l'économique, l'écologique et le culturel, sous l'objectif de parvenir à une croissance raisonnée respectueuse des populations comme de leur environnement. Or la figure de l'Anthropocène, associée d'ailleurs à celle de Gaïa ${ }^{80}$, comme les faces opposées d'une même pièce, en tant qu'elles pointent l'arrivée de l'humanité au pied de limites infranchissables (l'épuisement des ressources énergétiques) et le dépassement irréversible de seuils au-delà desquels les systèmes dont nous dépendons (climat, écosystèmes, biodiversité...) se dérèglent et ouvrent la perspective d'un avenir totalement imprévisible - ou d'un futur absent ${ }^{-81}$, est non seulement venue remettre en cause la perspective d'une croissance même durable ou verte mais en même temps aiguiser nos interrogations sur la possibilité de penser et de vivre un monde unifié, un monde aux ontologies reconfigurées. Cette perspective suppose une approche non restrictive de ce que nous nommons « environnement ${ }^{82}$ ». Désormais le mot ne peut se limiter à désigner des milieux ou des écosystèmes naturels avec lesquels l'homme, qu'il s'en perçoive extérieur ou intérieur, entretiendrait et ferait valoir une relation instrumentale. Dans la perspective ouverte par l'écologie de la perception ${ }^{83}$, l'environnement se définit par les prises ou les "affordances" qui assurent nos actions et nous façonnent; il se définit également par les relations, les transactions, les partenariat noués avec les non-humains de Bruno Latour ${ }^{84}$, les non-humain-e-s non-machines de Donna Haraway ${ }^{85}$, co-définissant un espace de coexistence et les modalités singulières de cette coexistence. Sous ces deux aspects qui mettent l'accent sur une relation d'accompagnement ou de compagnonnage, " la perception de l'environnement est un mode d'engagement dans le monde ${ }^{86}$ et c'est au sein de ce processus que se construisent mutuellement des personnes et leur 
environnement. L'intérêt de cette approche est qu'elle découple l'environnement de la nature - celui-là ne s'identifie pas à celle-ci - et justifie l'interrogation du partage natureculture, particulièrement féconde dans les travaux d'anthropologie, de sociologie ou de philosophie récents, rapidement regroupés sous le chapeau d'un « tournant ontologique ${ }^{87}$ ».

À quoi sert le patrimoine aujourd'hui ? Que peuvent être des « expériences patrimoniales du vivant" à l'heure de l'Anthropocène? Je pose la question de l'opérativité du patrimoine devant les problèmes et enjeux contenus dans cette désignation. Une première réponse est à lier au renversement de perspective avancé plus haut : dépassant l'objectif de représenter le passé, le patrimoine porterait souci de «renouer avec l'art de faire attention »- proposition empruntée à Isabelle Stengers ${ }^{88}-$, au sens où il pourrait contribuer à prendre le contre-pied « du droit de ne pas faire attention » qui s'est imposé au nom du progrès et de la croissance (ibid.) ? Cette proposition n'est sans faire écho à la proposition formulée par Yves Citton d'une écologie de l'attention ${ }^{89}$ - que pouvons-nous faire de notre attention? -, permettant de soutenir l'attention à la fois aux autres (aux formes de la différence) et aux défis environnementaux de notre temps. Les grands changements pointés par la désignation anthropocénique - climatique (réchauffement global, montée des océans, déplacement des populations), biologique (sixième extinction), chimique (acidification des océans), en même temps qu'une réévaluation de la place de l'humain dans le monde - son « retour sur Terre », pour suivre la proposition de Bruno Latour - donnent une acuité à la question du ou des héritages. Devant l'Anthropocène, une pensée du patrimoine devient une pensée de l'héritage : il s'agit d'interroger ce dont on hérite - non seulement des biens ou des êtres positifs, mais des lieux de mémoire de mort (Auschwitz), des espaces dégradés, des sites industriels abandonnés et pollués (parfois investis par artistes), des pertes irrémédiables (d'écosystèmes, d'espèces animales ou végétales), des déchets pullulants et des restes éternels (des résidus chimiques aux résidus nucléaires) -, mais aussi ce que l'on engage comme héritage potentiel, par le présent que l'on construit autant avec des gestes de dégradation et de destruction qu'avec des gestes de sauvegarde. Poser le patrimoine comme héritage est une manière d'en finir avec une forme d'innocence qui, comme l'avance Vinciane Despret ${ }^{90}$, consiste à dire « qu'on ne choisit pas ses ancêtres ». Choisir ses ancêtres, hériter, c'est au contraire, un, se rendre responsable de ce dont nous héritons, tout en travaillant, deux, à instaurer des choses et des êtres qui comptent (ce à quoi nous tenons), afin, trois, d'être comptable de ce que nous léguons. S'il est vrai qu'à l'âge du monument a succédé un âge du patrimoine, je propose de considérer la possibilité que, devant l'Anthropocène, se profile un âge de l'héritage.

\section{Participations citoyennes}

Pour autant, l'Anthropocène est une figure controversée. En fait, le mot ne satisfait personne mais fait parler tout le monde. Le débat, ouvert en particulier par la nouvelle histoire environnementale, porte sur la date à partir de laquelle faire débuter la nouvelle époque en même temps, laquelle a une incidence sur la détermination des responsabilités. Pour le dire vite, ces dernières sont-elles imputables à une humanité indifférenciée ? Ne doit-on pas plutôt incriminer une partie de l'humanité, celle qui a pris part à l'essor industriel, en Angleterre d'abord, puis dans d'autres pays occidentaux, et au développement du capitalisme? Ne faut-il pas préférer un autre terme qui soit plus en 
accord avec l'histoire du "monde du carbone »: Anglocène, Occidentalocène, Capitalocène...91 ? Par ailleurs, comme le souligne justement Bruno Latour, le mot pointe à la fois « une agency aux humains et son retrait» (« l'activité humaine est relayée par des forces que nous risquons de ne pas maitriser »). Mais lors, remarque Christophe Bonneuil ${ }^{92}$, le concept d'Anthropocène n'appartient-il pas au langage d'un nouveau géo-pouvoir qui met le sort de la planète entre les mains des scientifiques et obère la perspective d'une écologie politique émancipatrice ${ }^{93}$ ? Voulant dépasser ce débat entre humains (ou terriens), i.e. qui ne concernerait que l'espèce humaine, rejetant donc Anthropocène comme Capitalocène (à dépasser par l'instauration de nouvelles relations), Donna Haraway propose, avec Chthulucène ${ }^{94}$, de considérer les destins liés, mêlés, entrecroisés, embrouillés des « puissances biotiques et abiotiques de la terre ${ }^{95}$ ».

Ainsi, alors que, de diverses manières, la politique s'immisce dans un débat marqué par un questionnement sur le statut des êtres qui peuplent la Terre et leurs interactions, il y a lieu de se demander si le patrimoine pourrait contribuer à l'élaboration de récits alternatifs au grand récit dominant de l'Anthropocène (un récit naturaliste ${ }^{96}$ ), soit des récits qui remettent radicalement en question le modèle de la croissance et/ou qui s'élèvent contre les inégalités environnementales et la " néo-libéralisation » de la nature ${ }^{97}$ . Pour apprécier cette potentialité, là encore, il faut s'appuyer sur la singularité de l'âge $\mathrm{du}$ patrimoine et penser son possible dépassement. Comment nommer, désigner, comprendre le phénomène de débordement des institutions et des monopoles scientifiques (monopole de l'Histoire sur le passé, de l'ethno-anthropologie sur la culture) qui caractérise le patrimoine-aujourd'hui, dans un contexte de crise de la modernité et de sa mise en cause comme fiction impossible ${ }^{98}$ ? D'aucuns, prolongeant la réflexion de Michel de Certeau sur l'invention du quotidien, ont voulu y voir la marque d'un régime « ordinaire ${ }^{99}$ » ou " profane ${ }^{100}$ » qui se déploie contre ou à côté de l'institutionnel et du savant mis en œuvre dans les dispositifs légaux de la centralité étatique et nationale; cette tension a pu faire l'objet d'une formulation plus neutre, ainsi le couple official/ unofficial heritage proposé par Rodney Harrison ${ }^{101}$. Dans une veine proche, on a voulu considérer que la profusion patrimoniale, souvent fustigée comme prolifération, traduisait un engouement public pour le patrimoine - en témoigne le succès jamais démenti des Journées européennes du patrimoine - et toute une palette d'usages du patrimoine considérés comme autant d'indices de démocratisation: ainsi la notion de "valeur sociale du patrimoine ", qui a trouvé récemment un certain écho auprès des professionnels et des institutions du patrimoine, notamment grâce à la convention de Faro (2005), est venue, en un sens, prendre acte de la profusion tout en faisant cependant le départ entre une valeur intrinsèquement patrimoniale, généralement octroyée par les spécialistes (valeurs d'art et d'histoire, en premier lieu), et des valeurs ajoutées, résultant de "réceptions ", de saisies ou d'usages dans un espace ouvert au multiple. Dans cette perspective, les « nouveaux » usages du patrimoine sont bien à distinguer de l'opération administrative-scientifique qui fait le patrimoine, i. e. qui est censée instruire « la valeur patrimoniale $»$ du patrimoine.

Ces désignations et problématisations ne sont pas satisfaisantes: non seulement, elles sont implicitement hiérarchisantes et référées à un étalon administratif-scientifique, mais elles ne rendent compte ni de la singularité des expériences multiples et diverses situées hors des institutions du patrimoine ni de leur opérativité sociale: en d'autres termes, de leur politicité intrinsèque. En quoi le politique peut-il être un ressort de l'activité patrimoniale - non pas le politique en tant que ce qui relève de l'art de 
gouverner -, on voit bien en quoi les politiques du patrimoine, ou des monuments historiques, s'agissant de la France du XIX ${ }^{e}$ siècle, ont été des instruments d'un processus de « police " présidant à l'ordonnancement de fonctions et de places -, mais le politique en tant qu'agir dans « la présupposition de l'égalité » et dans la visée d'émancipation ${ }^{102}$ ? J'émets l'hypothèse d'une dimension citoyenne de l'engagement patrimonial: je n'envisage pas le débordement de l'activité patrimoniale hors de son régime d'action publique comme l'expression d'une simple démocratisation d'un idéal de culture - ce qui ne ferait que conforter une perspective top-down. Au contraire, sous le vocable « d'action, de contribution ou de participation citoyenne", je voudrais précisément interroger l'intensité de dynamiques bottom-up : dans quelle mesure et selon quelles modalités peuton dire que des actions patrimoniales s'apparentent à ces formes de mobilisation que l'on dit citoyennes, souvent protestataires et conflictuelles, voire portées par un militantisme radical ${ }^{103}$, qui se nouent sur des problèmes d'usages de l'espace, d'aménagement du territoire, de services publics ${ }^{104}$, de préservation de modes de vie et qui, ce faisant, interrogent et mettent à l'épreuve l'idéal et le fonctionnement démocratiques de nos sociétés?

Plus précisément, la participation citoyenne dans et par le patrimoine peut être envisagée selon deux pôles, l'un constitué par les formes de collaboration que des personnes ou des collectifs peuvent nouer avec les institutions patrimoniales (à vocation de connaissance, telle que l'Inventaire), l'autre par les actions qui se singularisent, s'affranchissent des autorités scientifiques ou institutionnelles. Le premier, appuyé sur la distinction amateur/professionnel, reproduit un schéma qui n'est pas sans rappeler le phénomène en plein essor des sciences dites «citoyennes" ou "participatives", reposant sur l'association d'amateurs aux spécialistes et aux scientifiques des sciences naturalistes de plein air, pour co-construire des connaissances ${ }^{105}$. Mais le patrimoine connait cependant une singularité qui échappe aux sciences citoyennes: l'activité patrimoniale peut s'exercer indépendamment ou à côté des institutions patrimoniales qui fonctionnent en régime scientifique ou en se déployant au sein de forums hybrides ou d'arènes délibératives. Aussi la deuxième manière de traiter d'une citoyenneté par le patrimoine vise-t-elle le dépassement de l'opposition amateur/professionnel en tant que cette opposition institue une autorité et par conséquent un pouvoir de disqualifier. Dire d'un patrimoine qu'il est citoyen ou d'une action patrimoniale qu'elle est citoyenne, c'est pointer la réelle singularité du geste: une problématisation, une prise de parole, une inventivité, une volonté de participation, de contestation, une action en commun, le surgissement d'un public (au sens de John Dewey) devant un problème à résoudre, etc., et considérer qu'elle a sa place à l'égal des gestes accomplis dans un cadre institutionnel.

L'exploration de cette dynamique, d'un pôle à l'autre, a pour corollaire une réflexion sur les formes de la démocratie participative ${ }^{106}$, le partage des savoirs et la reconnaissance d'une diversité de modes de connaissance. Pour autant, mettre en regard patrimoine et citoyenneté n'est pas exempt de risques et ce à un double titre. D'une part, au regard de l'ambivalence de la notion renvoyant d'ailleurs à l'ambivalence de "politique »: le/la politique crée des citoyens qui le/la cautionnent et le/la légitiment mais est aussi un lieu de construction de la citoyenneté entendue alors comme capacité. On comprendra que c'est moins la référence à la citoyenneté comme statut qui m'intéresse que la référence à une citoyenneté entendue comme « capacité » ou " pouvoir d'agir » en tant qu'« habitant de ». D'autre part, au regard de la caractérisation des savoirs, des compétences et de leurs détenteurs engagés, circulant et se confrontant dans les forums hybrides ou les nouvelles 
arènes de patrimonialisation: la définition «citoyenne» des actions ne lève pas automatiquement - comme le montrent des travaux sur «l'engagement citoyen ${ }^{107}-$ la tension entre un niveau savant ou expert et un niveau profane ou ordinaire, et donc ne questionne pas nécessairement le partage des savoirs et des expertises. Mon ambition est de saisir le seuil à partir duquel l'idée de citoyenneté permet de penser le « devenir sujet politique » et l'émancipation. Dès lors, je ne vise pas la validation d'une hypothèse mais bien sa mise à l'épreuve : il s'agit d'inscrire l'anthropologie des formes citoyennes de la patrimonialisation dans la voie ouverte d'une anthropologie de la citoyenneté fondée sur " une exploration empirique et une pensée “des citoyennetés" dans leur complexité et leur diversité108 $»$.

\section{Habiter le temps}

«Un territoire, a écrit Bruno Latour ${ }^{109}$, c'est d'abord la liste des entités dont on dépend ». S'il est vrai que son habitabilité tient aux épreuves que constituent les ajouts ou les retraits de la liste, alors, ajoutait-il, voilà un terme qui « dépend du temps bien plus que de l'espace ». Procédures d'ajouts et de constitution de listes, les politiques du patrimoine ont depuis longtemps souligné cette dimension temporelle de l'espace territorial. L'irruption de l'Anthropocène - événement qui fait événement - vient donner un tour nouveau à cette relation consubstantielle entre le temps et le territoire quand il apparaît que le territoire est la Terre et que la somme des pertes risque de finir par l'emporter sur la somme des gains. Interroger notre possibilité de continuer d'habiter la Terre consiste à évaluer, à développer notre capacité à « habiter le temps ». Cette question a été portée de manière assez pionnière par l'historien et militant politique Jean Chesneaux ${ }^{110}$ : quelques années avant François Hartog ${ }^{111}$ et de manière plus incisive et radicale, il déplorait que « la valorisation présentéiste de l'immédiat » contribuât à la décomposition de la relation fondatrice entre le passé, le présent et le futur ${ }^{112}$. Et de plaider pour une culture politique $\mathrm{du}$ temps, fondée sur la conception d'un «temps-compagnon » et passant par l'affirmation de l'autonomie et de la capacité propre du temps social, qu'il définissait comme un "temps citoyen » face au «temps du Marché » et au "temps de l'État »"113. Vingt ans plus tard, ce plaidoyer est plus que jamais d'actualité. L'orientation de recherche que je propose sur l'opérativité de l'idée patrimoine devant la perspective inédite contenue dans la figure de l'Anthropocène suppose, ainsi que je l'ai avancé, de redimensionner la question du patrimoine sous les topiques de l'héritage, du vivant et du commun, et par conséquent de la replacer devant son horizon, soit l'institution de politiques du temps, soit l'agencement des liens entre les termes de la chaîne temporelle. La question vive est alors: quels patrimoines pour quelle politique du temps devant l'Anthropocène?

L'approche pragmatiste que je défends considère la profonde ambivalence du patrimoine au regard de la variété des formes qu'il prend selon qu'il est entre les mains de l'État, de collectivités territoriales, de communautés, d'associations ou de tour-operators: il peut être autant un instrument de gouvernementalité, i.e. de discipline ou de contrôle, que l'expression d'une prise de parole, le moyen de développement d'un espace public (au sens d'Habermas), ou d'un public (au sens de J. Dewey), un lieu de résistance à la domination, une brèche dans les rentes de monopole capitaliste ${ }^{114}$ et une arme critique. C'est cette dimension alternative et critique qui est privilégiée avec la proposition d'une «patrimonialité citoyenne». En ce sens, cette approche procède du souci 
anthropologique d'accorder un traitement égal et non disqualifiant à ces actions qui débordent la raison patrimoniale institutionnelle : elles sont à prendre au sérieux comme sont à prendre au sérieux les formes d'expertise qu'elles suscitent ou les modes de connaissance sur lesquels elles s'appuient, considérés comme distincts de l'expertise ou $\mathrm{du}$ mode de connaissance scientifique. En somme, je vise moins une anthropologie du patrimoine qu'une anthropologie des connexions et des interactions qui se nouent sous ce nom comme sous d'autres - monument, mémoire, culture, tradition, territoire, etc. -, des mobilisations qui s'y jouent, des causes qui s'y défendent. C'est la condition pour que soit pleinement saisi le rapport du cognitif au politique. Pour autant, mon interrogation de la potentialité critique du patrimoine vise in fine la pertinence de l'entrée par le patrimoine. La question a déjà été posée à la culture par Nancy Fraser dans le débat qu'elle a engagé avec Axel Honneth autour du paradigme de la reconnaissance ${ }^{115}$, et la recherche de sa conciliation avec le paradigme de la redistribution ${ }^{116}$. Est-il possible, dans un même ordre d'idée, de dépasser la réserve fréquemment adressée au patrimoine selon laquelle il serait une modalité faiblement compensatoire? Est-il envisageable d'obvier aux effets de neutralisation et de mise à distance qui seraient inhérents à toute patrimonialisation ? Voire est-il permis de conjurer le principe de charité qui semble animer un souci patrimonial de reconnaissance paternelle des démunis, des exclus, des invisibles et des oubliés? Le concept de patrimoine peut-il alors contribuer à nourrir ou à renouveler l'action politique?

De ce point de vue, l'hypothèse des patrimoines-héritages contient l'instauration d'un espace de controverse avec tout ce qui ressortit à une conception orthodoxe du patrimoine. Sans doute y a-t-il un enjeu politique à affranchir ce mot piégé des significations dominantes; sans doute est-il nécessaire de le délester de la charge de normativité inhérente à deux siècles de célébration de la culture légitime, du monument national au patrimoine de l'humanité. Le libérer également de sa teneur eurocentrée et de la profession de foi universaliste guère coûteuse qui l'accompagne. Via l'idée d'un patrimoine-héritage, les expérience citoyennes du vivant, transmis et à transmettre, loin d'une universalité posée d'emblée et comme réponse à la crise écologique, sont à la fois de plus modeste envergure et plus radicales en ce qu'elles visent « une réhabilitation du lieu et des liens ${ }^{117}$ ", sont portées par l'idée "d'un monde fait d'une pluralité de mondes ${ }^{118}$ ", sont sensibles, comme le montre le perspectivisme amérindien ${ }^{119}$, à la possibilité d'accueillir en partenaires ou en entités-compagnes des " êtres possibles 120 ", et au final travaillent le dépassement du dualisme nature/culture. De ce point de vue, elles ouvriraient sur l'instauration de choses compagnes nourrissant l'idée d'un patrimoinecompagnon: dépassant le balancement entre l'être et l'avoir pour un «être avec ». Plus radicales également en ce qu'elles remettent en cause nommément le capitalisme, système global qui organise et structure notre réalité, et visent à se libérer de sa tyrannie et se prémunir de sa toxicité : en somme, le capitalisme comme la désignation expresse d'une modernité qui s'est cristallisée en Europe au XVIII ${ }^{e}$ siècle et a étendu sa raison sur la totalité des formes de vie de notre planète au terme d'une triple conquête : économique, écologique et culturelle ${ }^{121}$. Expériences, encore, aux tonalités ethniques ou sociales qui, ici ou ailleurs, explorent et portent des projets alternatifs à la modernité néo-libérale, voire une sortie radicale de la modernité - en y négociant, et même reconstruisant, dans l'action et en toute localité, l'articulation ou l'intrication de ces trois «ordres" (l'économique, l'écologique et le culturel) trop commodément distingués. Expériences, enfin, qui proposent des futurs et des passés moins imaginés, imaginaires ou utopiques que construits dans l'action, moins des utopies comme horizon d'attente que des 
"utopies réelles », moins des projections idéales et lénifiantes que des gestes concrets qui, tout en prenant au sérieux la proposition "anthropocénique», travaillent à questionner, à explorer et à revendiquer la démocratie: démocratie "réelle ${ }^{122}$ ", démocratie radicale ${ }^{123}$, démocratie aux marges du monde occidental ${ }^{124}$.

\section{NOTES}

1. - HARVEY, David. Villes rebelles. Du droit à la ville à la révolution urbaine. Paris : Buchet Chastel, 2015, p. 170.

2. - HARAWAY, Donna. "Sympoièse, sf, embrouilles multispécifiques ». Dans DEBAISE, Didier et STENGERS, Isabelle (dir.). Gestes spéculatifs. Dijon : Les Presses du réel, 2015, p. 72.

3. - HERTZ, Ellen et CHAPPAZ-WIRTHNER, Susan (dir.). «Ethnographies des pratiques patrimoniales : temporalités, territoires, communautés ». Ethnographiques.org, juillet 2012, $\mathrm{n}^{\circ} 24$.

4. - Le phénomène ne concernerait pas seulement la France, mais aussi l'espace européen, voire le monde anglo-saxon. Voir sur ce point: HARRISON, Rodney. Heritage. Critical approaches. Abingdon/New York : Routledge, 2013.

5. - Cet essai reprend et développe l'argument central d'un projet que j'ai coordonné et présenté en 2014 dans le cadre de l'appel à projet générique de l'ANR, projet dans lequel je posais et souhaitais interroger l'idée de "patrimoine citoyen»: Des patrimoines citoyens? Actualité de l'activité patrimoniale : une mise en perspective internationale. Malheureusement, il n'a pas été retenu en sélection finale. La qualification "citoyenne" d'actions patrimoniales me paraît toujours justifiée quand en même temps elle peut trouver sa limite dès lors que l'on souhaite s'engager dans la voie d'une anthropologie patrimoniale « réellement politique». Aussi, ce texte, écrit début 2016, a été suivi de versions ou de textes en cours de publication qui font état de cette sorte d'insatisfaction que suscite le mot "citoyen»: TORNATORE, J.-L. "Questions de démocratie patrimoniale ». Dans AUCLAIR, Elizabeth, HERZOG, Anne, POULOT, Marie-Laure (dir.). «De la participation à la co-construction des patrimoines urbains : l'invention du commun?». Éditions le Manuscrit, 2017 (sous presse); Id. «Patrimoines et citoyenneté. Considérations actuelles ». Dans BOTEA, Bianca, POPESCU, Dana (dir.). Citoyenneté et diversité : lieux, pratiques et discours actuels . Presses universitaires de Lyon (à paraître).

6. - Sur ce point, je renvoie à mon article : TORNATORE, Jean-Louis. «L'espace de la mémoire, une approche anthropologique ou comment dépasser le concept de "lieu de mémoire" ». Dans MAJERUS, Benoît, KMEC, Sonia, MARGUE, Michel (dir.). Dépasser le cadre national des "Lieux de mémoire ». Innovations méthodologiques, approches comparatives, lectures transnationales / Nationale Erinnerungsorte hinterfragt. / Methodologische Innovationen, vergleichende Annäherungen, transnationale Lektüren. Bruxelles : Peter Lang, 2009, p. 33-48.

7. - J'ai proposé une problématisation du couple mémoire-patrimoine dans TORNATORE, J.-L. «Les banquets de la mémoire à l'auberge du patrimoine ». Dans Id. (dir.). L'Invention de la Lorraine industrielle. Quêtes de reconnaissance, politiques de la mémoire. Paris: Riveneuve éditions, 2010, p. 5-62.

8. - LENIAUD, Jean-Michel. L'Utopie française. Essai sur le patrimoine. Paris: Mengès, 1992 ; Id. Chroniques patrimoniales. Paris : Norma, 2001.

9. - LENIAUD, Jean-Michel. "Voyage au centre du patrimoine». Dans FABRE, Daniel (dir.). Domestiquer l'histoire. L'ethnologie des monuments historiques. Paris : Éd. de la MSH, 2001, p. 181. 
10. - Ibid., p. 182.

11. - Id. Chroniques patrimoniales... Op. cit., p. 98.

12. - Voir le commentaire de Noël Barbe : BARBE, Noël. «Actions patrimoniales ». Les Nouvelles de l'Archéologie, 2003, n 93, p. 5.

13. - Voir l'article qu'il publiait en 1987 dans la revue Terrain sous un pseudonyme: OLLIVIER, Eugène. «Les monuments historiques demain... ». Terrain, 1987, nº 9, p. 124-127.

14. - LENIAUD, Jean-Michel. « Voyage au centre du patrimoine ». Art. cit., p. 183.

15. - NORA, Pierre. «Entre mémoire et histoire. La problématique des lieux ». Dans Id. (dir.). Les Lieux de mémoire. Vol. 1, La République [1984]. Paris : Gallimard, 1997, p. 28.

16. - FABRE, Daniel. "Ethnologie et patrimoine en Europe. Conclusions et perspectives du colloque de Tours ». Terrain, 1994, n 22, p. 148 (article repris dans Id. (dir.). L'Europe entre cultures et nations. Paris : Éd. de la MSH, 1996, dont il constitue l'introduction).

17. - Ibid., p. 148.

18. - «Ethnologie et patrimoine en Europe. Identité et appartenance, du local au supra national », Tours, 8-11 décembre 1993. Les actes de ce colloque ont été publiés dans L'Europe entre cultures et nations. Op. cit.

19. - En somme, la culture entre guillemets selon Manuela Carneiro da Cunha : CUNHA, Manuela Carneiro da. Savoir traditionnel, droits intellectuels et dialectique de la culture [2006]. Paris : Éd. de l'Éclat, 2010. L'argument est au cœur de la fondation du Laboratoire d'anthropologie et d'histoire de l'institution de la culture (LAHIC); voir FABRE, D. L'Institution de la culture. Pour une anthropologie comparée, Projet d'unité mixte de recherche. Paris: juin 2000, 36 p. dactyl.; Id. «L'institution de la culture : naissance d'un laboratoire ». Culture \& recherche, oct.-nov. 2001, $\mathrm{n}^{\circ}$ 87, p. 6.

20. - FABRE, Daniel. « Le patrimoine, l'ethnologie». Dans NORA, Pierre (dir.). Science et conscience du patrimoine. Paris : Fayard/Éd. du patrimoine, coll. « Actes des Entretiens du patrimoine », 1997, p. 59-72.

21. - RAUTENBERG, Michel. "L'intervention ethnologique. Témoignage et éléments de réflexion sur les relations entre recherche et action culturelle dans une direction régionale des affaires culturelles». Dans POIRRIER, Philippe et VADELORGE, Loïc. Pour une histoire des politiques du patrimoine. Paris: Comité d'histoire du ministère de la Culture/Fondation de la Maison des sciences de l'homme, 2003, p. 469-489.

22. - RAUTENBERG, Michel. La Rupture patrimoniale. Bernin : À la croisée, 2003.

23. - BARBE, Noël (dir.). Dossier «Actions patrimoniales ». Les Nouvelles de l'Archéologie, 2003, $\mathrm{n}^{\circ}$ 93.

24. - TORNATORE, Jean-Louis. « Le patrimoine comme objet-frontière ». Dans De la connaissance à la gestion du patrimoine. Actes des journées rencontres entre parcs naturels régionaux de France et services de la direction de l'Architecture et du Patrimoine, La Roche-Guyon, 17-18 mars 1999. Paris : Fédération des PNR/ministère de la Culture/ministère de l'Aménagement du territoire et de l'Environnement, 2000, p. 21-24.

25. - TRÉPOS, Jean-Yves. La Sociologie de l'expertise. Paris: PUF, 1996; «L'expertise comme équipement politique de la société civile ». Questions de communication, 2002, n 2, p. 7-18 (en ligne sur Revues.org https://questionsdecommunication.revues.org/7064 [consulté le 11/10/2017]). La pragmatique de l'expertise qu'il a mise en œuvre repose sur l'idée que l'expertise relève moins de la profession que de la position : en d'autres termes, ce n'est pas la profession qui fait l'expert, on n'est jamais que reconnu comme expert : c'est une position attribuée qui est sans cesse mise en doute, à défendre ou bien une position auto-attribuée qui est à conquérir.

26. - TORNATORE, Jean-Louis. «Les formes d'engagement dans l'activité patrimoniale. De quelques manières de s'accommoder au passé ». Dans MEYER, Vincent et WALTER, Jacques (dir.). Formes de l'engagement et espace public, Questions de communication, série actes 3. Nancy: Presses universitaires de Nancy, 2006, p. 515-538. 
27. - Id. « Beau comme un haut fourneau. Sur le traitement en monument des restes industriels ». L'Homme, $\mathrm{n}^{\circ}$ 170, 2004, p. 79-116; Id. "L'invention de la Lorraine industrielle. Note sur un processus en cours ». Ethnologie française, 2005/4, XXXV, p. 679-689 ; Id. « La reconnaissance pour mémoire. Un parcours dans l'espace de la mémoire de la "Lorraine sidérurgique»». Dans TORNATORE, Jean-Louis. (dir.). L'Invention de la Lorraine industrielle. Op. cit., p. 161-191.

28. - Ce paragraphe s'appuie sur une réflexion menée avec Noël Barbe dans le cadre d'un colloque sur ce thème, «Du moment du patrimoine ethnologique » qui s'est tenu à Dijon du 7 au 9 décembre 2016.

29. - FABRE, Daniel. «Le patrimoine porté par l'émotion ». Dans Id. (dir.). Émotions patrimoniales. Paris : Éd. de la MSH, 2013, p. 17. Une première occurrence du motif se trouve en introduction de FABRE, Daniel. L'Europe entre culture et nations. Op. cit.

30. - SCHAER, Roland. Art. «Patrimoine». Dans CANTO-SPERBER, Monique (dir.). Dictionnaire d'éthique et de philosophie morale. Paris : PUF, 1996, p. 1090.

31. - Ibid.

32. - GUILLAUME, Marc. La Politique du patrimoine. Paris : Galilée, 1980, p. 139.

33. - Une version prototypique de Concorde est exposée depuis 1973 au musée de l'Air et de l'Espace, au Bourget.

34. - Ibid., p. 140.

35. - Voir par exemple RAUTENBERG, Michel, MICOUD, André, BÉRARD, Laurence, et al. (dir.). Campagnes de tous nos désirs. Patrimoines et nouveaux usages sociaux. Paris : Éd. de la MSH, 2000.

36. - L'ouvrage remarqué de Hervé GLÉVAREC et Guy SAEZ. Le Patrimoine saisi par les associations. Paris : la Documentation française, 2002, donne un bon aperçu de ce caractère d'espace public, c'est-à-dire, au sens habermassien, d' " arène institutionnalisée d'interaction discursive " (FRASER, Nancy. Qu'est-ce que la justice sociale? Paris: La Découverte, 2005, p. 109) : la notion circule, fait faire, est travaillée dans les actions associatives, est commentée comme mobile justificatif, etc.

37. - BERGER, Alain, CHEVALIER, Pascal, CORTÈS, Geneviève, et al. «Patrimoines, héritages et développement rural en Europe ». Dans Ibid. (dir.). Patrimoines, héritages et développement rural en Europe. Paris : L'Harmattan, 2010, p. 5.

38. - Voir FRANÇOIS, Hugues, HIRCZAK, Maud et SENIL, Nicolas. « La dimension patrimoniale des ressources territoriales. Quel rôle dans les trajectoires de développement?». Dans BERGER, Alain, CHEVALIER, Pascal, CORTES, Geneviève, et al. Op. cit., p. 20-44.

39. - HIRCZAK, Maud, et al. «Le modèle du panier de biens. Grille d'analyse et observation de terrain ». Économie rurale, 2008, $\mathrm{n}^{\circ} 308, \mathrm{p}$. 58. « Le panier combine un ensemble de caractéristiques objectives des produits (goût, senteur, saveur...) à un ensemble d'attributs, d'images ou de symboles ayant un caractère cognitif. Les produits du panier, qui résultent d'une culture et d'une histoire longue, se renvoient les uns aux autres, avec une origine territoriale commune et une image de qualité cohérente » (ibid.).

40. - FRANÇOIS, Hugues, et al., Art. cit., p. 33.

41. - MARIS, Virginie. Nature à vendre. Les limites des services écosystémiques. Versailles : Éd. Quae, 2014, p. 29.

42. - Lequel reconnaît aujourd'hui : «On ne peut pas se libérer de l'injonction patrimoniale, elle est maintenant trop engagée dans l'économie du tourisme » (GUILLAUME, Marc. « L'impérialisme mou de la culture », entretien avec Bernard Jeannot et Frédérique Le Graverend. Area, 2011, n 25, p. 20).

43. - FORTUNET, Françoise. "Patrimoine et identité : approches juridiques ». Dans BARRÈRE, Christian, BARTHÉLÉMY, Denis, NIEDDU, Martino, et al. (dir.). Réinventer le patrimoine. De la culture à l'économie, une nouvelle pensée du patrimoine ? Paris : L'Harmattan, 2005, p. 79.

44. - BOLTANSKI, Luc et ESQUERRE, Arnaud. «La "collection", une forme neuve du capitalisme. La mise en valeur économique du passé et ses effets ». Les Temps modernes, 2014/3, p. 5-72. 
45. - Ibid., p. 59.

46. - Voir en particulier le chapitre intitulé «L'art de la rente». Dans HARVEY, David. Villes rebelles... Op.cit., p.169-205. Également, Id. Géographie de la domination. Paris: Les Prairies ordinaires, 2008.

47. - Id. Villes rebelles... Op. cit., p. 200.

48. - Ibid., p. 202.

49. - Ibid., p. 203.

50. - FRESSOZ, Jean-Baptiste, GRABER, Frédéric, LOCHER, Fabien et QUENET, Grégory. Introduction à l'histoire environnementale. Paris : La Découverte, 2014 ; CHARTIER, Denis et RODARY, Estienne (dir.). Manifeste pour une géographie environnementale. Paris: Presses de Sciences Po, 2015. Les directeurs de ce livre-manifeste écrivent en introduction: "On entend poser les jalons d'une géographie, non pas de l'environnement comme objet que surplomberait la discipline, mais environnementale en tant qu'elle est elle-même travaillée et transformée par cet adjectif » («Géographie, écologie, politique : un climat de changement », p. 17).

51. - En particulier sous l'impulsion de Daniel Fabre: voir FABRE, Daniel. «L'ordinaire, le familier, l'intime, loin du monument ». Dans HOTTIN, Christian et VOISENAT, Claudie (dir.). Le Moment patrimonial. Mutations contemporaines des métiers du patrimoine. Paris : Éd. de la MSH, 2016.

52. - FABRE, Daniel. « Le patrimoine porté par l'émotion ». Art. cit., p. 20.

53. - HARAWAY, Donna. «Savoirs situés: la question de la science dans le féminisme et le privilège de la perspective partielle ». Dans Des singes, des cyborgs et des femmes. La réinvention de la nature. Paris/Arles : Jacqueline Chambon/Actes Sud, 2009, p. 323-352.

54. - Le conseiller à l'ethnologie était-il un chercheur? Une réponse succincte et définitive à cette surprenante question posée par un «lecteur » se trouve dans BARBE, Noël, TORNATORE, Jean-Louis. «Pour une anthropologie plébéienne et pragmatiste du patrimoine». In Situ. Sous le regard des Sciences Sociales, à paraître.

55. - TORNATORE, Jean-Louis. «La difficile politisation du patrimoine ethnologique ». Terrain, $2004, n^{\circ} 42$, p. $149-160$.

56. - COUTELLEC, Léo. La Science au pluriel. Essai d'épistémologie pour les sciences impliquées. Versailles : Éditions Quae, 2015.

57. - Ces topiques sont explorées dans TORNATORE, Jean-Louis. « L'esprit de patrimoine ». Terrain , 2010, n 55, p. 106-127.

58. - Cette formule, que j'ai appliquée au patrimoine dans la recherche d'une formulation générique, a pour origine la publication concomitante de deux textes. L'ouvrage d'Émilie HACHE. Ce à quoi nous tenons. Propositions pour une écologie pragmatique. Paris : La Découverte, 2010, et la présentation de la traduction de la Formation des valeurs de John Dewey : BIDET, Alexandra, QUÉRÉ , Louis et TRUC, Jérôme ("Ce à quoi nous tenons. Dewey et la formation des valeurs", introduction à DEWEY, John. La Formation des valeurs. Paris : La Découverte, 2011, p. 5-64). «Ce à quoi nous tenons" est un motif pragmatiste dont la formulation coïncide avec mon souci d'élaborer une approche pragmatiste du patrimoine.

59. - TORNATORE, Jean-Louis. « Words for Expressing What We Care About. The Continuity and the Exteriority of the Heritage Experience ». Dans BONDAZ, Julien, GRAEZER BIDEAU, Florence, ISNART, Cyril, et al. (dir.). Les Vocabulaires locaux du "patrimoine». Traductions, négociations, transformations. Zürich : Lit Verlag, 2014, p. 31-54.

60. - JAMES, William. Essais d'empirisme radical [1912]. Marseille : Agone, 2005.

61. - DEWEY, John. «Le postulat de l'empirisme immédiat» [1905]. Critique, 2012/12, nº 787, Retour à Dewey, p. 1014-1025 ; Id. Expérience et nature [1929]. Trad. Joëlle Zask. Paris : Gallimard, 2012.

62. - LATOUR, Bruno. Enquête sur les modes d'existence. Une anthropologie des modernes. Paris: La Découverte, 2012, p. 69. 
63. - Sa spectacularisation autant que sa marchandisation, sous la forme par exemple de l'histoire vivante (Living History).

64. - Telle celle que peut proposer la Maison des cultures du monde.

65. - MICOUD, André. " Musée et patrimoine : deux types de rapport aux choses et au temps». Hermès, 20, 1996, p. 115-123 ; Id. «La patrimonialisation ou comment dire ce qui nous relie (un point de vue sociologique) ». Dans BARRÈRE, Christian, BARTHÉLÉMY, Denis, NIEDDU, Martino, VIVIEN, Franck-Dominique (dir.). Réinventer le patrimoine. Op. cit., p. 81-96.

66. - MICOUD, André. « La patrimonialisation... ». Art. cit., p. 87.

67. - Ibid., p. 88.

68. - Ibid., p. 85. Je pourrais faire la même remarque quant aux topiques que j'ai proposées.

69. - Voir BOOKCHIN, Murray. Qu'est-ce que l'écologie sociale? [1982]. Lyon : Atelier de création libertaire, 2012; Id. "Écologie sociale vs écologie profonde: un défi pour le mouvement écologiste ». Dans HACHE, Émilie (dir.). Écologie politique. Cosmos, communautés, milieux [1987]. Paris : Éd. Amsterdam, 2012, p. 171-181 ; DI CHIRO, Giovanna. «La nature comme communauté. La convergence de l'environnement et de la justice sociale » [1996]. Dans ibid., p. 121-153.

70. - J'avais souligné ce point dès 2007 dans une communication («Le patrimoine culturel immatériel. Une nouvelle catégorie entre action publique et anthropologique?») publiée en 2011 : « Du patrimoine ethnologique au patrimoine culturel immatériel : suivre la voie politique de l'immatérialité culturelle ». Dans BORTOLOTTO, Chiara (dir.). Le Patrimoine culturel immatériel. Enjeux d'une nouvelle catégorie. Paris : Éd. de la MSH, 2011, p. 213-232.

71. - Selon la formule choc de Christian Jacquelin, «Le patrimoine culturel immatériel, c'est le patrimoine ethnologique sans les ethnologues ».

72. - BOLTANSKI, Luc et ESQUERRE, Arnaud. Art. cit.

73. - TORNATORE, Jean-Louis. « Retour d'anthropologie : le "Repas gastronomique des Français". Éléments d'ethnographie d'une distinction patrimoniale». Ethnographiques.org, nº 24, 2012 (en ligne).

74. - DI CHIRO, Giovanna. Art. cit., p. 138.

75. - GUILLAUME, Marc. La Politique du patrimoine. Op. cit.

76. - Voir, par exemple, dans une orientation éco-féministe et altermondialiste : STARHAWK. Rêver l'obscur. Femmes, magie et politique. Paris : Cambourakis, 2015. Voir également la critique de la théorie du deuil de Vinciane DESPRET. Au bonheur des morts. Récits de ceux qui restent. Paris: La Découverte, 2015.

77. - Le rapport entre patrimoine et Anthropocène, pour le dire vite, a fait l'objet d'une journée d'étude que j'ai organisée avec Anne-Sophie Haeringer en septembre 2015: «Actualité du patrimoine à l'Anthropocène. Ontologies et politiques", Dijon, Centre Georges-Chevrier, 17-18 septembre 2015 (publication des actes en cours).

78. - Parmi une production qui commence à être abondante : BONNEUIL, Christophe et FRESSOZ, Jean-Baptiste. L'Événement anthropocène. La Terre, l'histoire et nous. Paris : Éd. du Seuil, 2013 ; SINAÏ, Agnès (dir.). Penser la décroissance. Politiques de l'Anthropocène. Paris : Presses de Sciences Po, 2013 ; Ead. L'Économie de l'après-croissance. Politiques de l'Anthropocène II. Paris : Presses de Sciences Po, 2015 ; HACHE, Émilie (dir.). De l'univers clos au monde infini. Paris : Éd. Dehors, 2014 ; LATOUR, Bruno. Face à Gaïa. Huit conférences sur le nouveau régime climatique. Paris : La Découverte, 2015. Le colloque qui s'est tenu au collège de France, peu avant la COP 21 (décembre 2015), offrait une bonne photographie de la mobilisation des sciences sociales et la manière dont elles ont investi la question: «Comment penser l'Anthropocène? Anthropologues, philosophes et sociologues face au changement climatique », Paris, 5-6 novembre 2015.

79. - Portail des humanités environnementales: http://humanitesenvironnementales.fr/ [consulté le 11/10/2017].

80. - LOVELOCK, James. La Terre est un être vivant. L'hypothèse Gaïa [1979]. Paris : Flammarion, 1993. 
81. - SERVIGNE, Pablo et STEVENS, Raphaël. Comment tout peut s'effondrer. Petit manuel de collapsologie à l'usage des générations présentes. Paris : Éd. du Seuil, 2015.

82. - INGOLD, Tim. The Perception of the environment: essays on livelihood, dwelling and skill. New York : Routledge, 2000.

83. - GIBSON, James. Approche écologique de la perception visuelle [1979]. Bellevaux : Éd. Dehors, 2014.

84. - LATOUR, Bruno. Enquête... Op. cit.

85. - HARAWAY, Donna. Des singes, des cyborgs et des femmes. Op.cit.; Ead. «Les promesses des monstres : politiques régénératives pour d'autres impropres/inaproprié-e-s ». Dans DORLIN, Elsa et RODRIGUEZ, Eva (dir.). Penser avec Donna Haraway [1993]. Paris : PUF, 2012, p. 158-229.

86. - INGOLD, Tim. Marcher avec les dragons. Bruxelles : Zones sensibles, 2013, p. 137.

87. - Id., The Perception of the environment... Op. cit. ; DESCOLA, Philippe. Par-delà nature et culture. Paris : Gallimard, 2005; CASTRO, Eduardo VIVEIROS de. Métaphysiques cannibales. Paris : PUF, 2009 ; BLASER, Mario. "Ontological Conflicts and the Stories of Peoples in Spite of Europe. Toward a Conversation on Political Ontology». Current Anthropology, vol. 54, $\mathrm{n}^{\circ}$ 5, 2013, p. 547-568; «L'anthropologie et le tournant ontologique ». Tsantsa, n²0, 2015.

88. - STENGERS, Isabelle. Au temps des catastrophes. Résister à la barbarie qui vient. Paris: La Découverte/Les Empêcheurs de penser en rond, 2009.

89. - CITTON, Yves. Pour une écologie de l'attention. Paris : Éd. du Seuil, 2014.

90. - DESPRET, Vinciane. «En finir avec l'innocence. Dialogue avec Isabelle Stengers et Vinciane Despret». Dans DORLIN, Elsa et RODRIGUEZ, Eva (dir.). Penser avec Donna Haraway. Op. cit., p. 22-45.

91. - MOORE, Jason W. "The Capitalocene, Part I: On the Nature and Origins of Our Ecological Crisis ", The Journal of Peasant studies, 2017, 44/3, p. 594-630 ; " Part II : Abstract Social Nature and the Limits to Capital », 2014, disponible en ligne : http://www.jasonwmoore.com/Essays.html [consulté le 11/10/2017]; BONNEUIL, Christophe. "Anthropocène, occidentalocène ou capitalocène? ». Magazine des Digital Studies, 2015, $\mathrm{n}^{\circ}$ 79, p. 22-29.

92. - Ibid.

93. - Cette thèse est développée dans le livre tout récent de Frédéric NEYRAT. La Part inconstructible de la Terre. Critique du géo-constructivisme. Paris: Éd. du Seuil, 2016. Dans une critique qui vise "Latour et ses alliés post-environnementalistes ", ce dernier dénonce les effets pervers du mythe de l'Anthropocène en ce qu'il postule qu'il revient à l'homme de réparer ce qu'il a fait, avec les mêmes moyens, au lieu précisément de remettre en question radicalement ces moyens.

94. - D'après Cthulhu, nom d'un monstre humanoïde à tête de seiche créé par Lovecraft dans une nouvelle publiée en 1928. D. Haraway fait de «Chthulu » une créature chtonienne élémentaire : elle donnerait alors son nom "à un temps qui a été, qui est toujours et qui pourrait encore être » («Sympoièse, sf, embrouilles multispécifiques »... Art. cit., p. 43). Il s'agit pour elle de penser.

95. - HARAWAY, Donna. «Sympoièse, sf, embrouilles multispécifiques »... Art. cit., p. 70. Voir également: "Anthropocène, Capitalocene, Plantationocene, Chthulucene: Making Kin». Environmental Humanities, 2015, vol. 6, p. 159-165.

96. - BONNEUIL, Christophe. « Anthropocène... ». Art. cit.

97. - Tel est le sens de "devant », dans le sous-titre. Il s'agit de tenir compte des controverses et critiques que suscitent le mot et de ne pas se livrer pieds et poings liés au récit qu'il légitime.

98. - LATOUR, Bruno. Nous n'avons jamais été modernes. Essai d'anthropologie symétrique. Paris: La Découverte, 1991.

99. - ISNART, Cyril. «Les patrimonialisations ordinaires. Essai d'images ethnographiées». ethnographiques.org, 2012, $\mathrm{n}^{\circ} 24$ (en ligne).

100. - BORTOLOTTO, Chiara. «Le trouble du patrimoine culturel immatériel ». Dans Ead. (dir.). Le Patrimoine culturel immatériel. Op. cit., p. 21-43. 
101. - HARRISON, Rodney. Heritage. Critical Approaches. Op. cit.

102. - RANCIÈRE, Jacques. Le Spectateur émancipé. Paris : La Fabrique éditions, 2008.

103. - On pense bien sûr au Comité invisible. À nos amis. Paris : La Fabrique éditions, 2014 ; voir aussi les publications du collectif Mauvaise Troupe : Constellations. Trajectoires révolutionnaires du jeune $21^{e}$ siècle. Paris : Éd. de l'Éclat, 2014 ; Défendre la Zad. Paris : Éd. de l'Éclat, 2016.

104. - FORET, Catherine. Quand les mobilisations citoyennes interpellent l'action publique. Les conflits territoriaux comme " apparitions de la démocratie ». Lyon : FRV 100/Communauté urbaine du Grand Lyon, 2014.

105. - CHARVOLIN, Florian, MICOUD, André et NYHART, Lynn K. (dir.). Des sciences citoyennes ? La question de l'amateur dans les sciences naturalistes. La Tour-d'Aigues : Éd. de l'Aube, 2007 ; ROUX, Jacques, CHARVOLIN, Florian, DUMAIN, Aurélie (dir.). Passions cognitives. L'objectivité à l'épreuve du sensible. Paris : Éd. des Archives contemporaines, 2013.

106. - Politix. Revue des sciences sociales du politique, vol. $15, \mathrm{n}^{\circ} 7,2002$; BLONDIAUX, Loïc et SINTOMER, Yves (dir.). Démocratie et délibération; BLONDIAUX, Loïc. Le Nouvel esprit de la démocratie. Actualité de la démocratie participative. Paris : Éd. du Seuil, 2008 ; BACQUÉ, Marie-Hélène et SINTOMER, Yves (dir.). La Démocratie participative. Histoire et généalogie. Paris: La Découverte, 2011.

107. - JONES, Kevin, et IRVIN, Alan. « Un espace d'engagement citoyen ? La participation profane et le changement institutionnel dans la gouvernance contemporaine des risques». Revue d'anthropologie des connaissances, 2013/1, vol. 7, n 1, p. 145-171.

108. - GAGNÉ, Natacha et NEVEU, Catherine . "L'anthropologie et la "fabrique" des citoyennetés». Anthropologie et Sociétés, 2009, vol.33, n², p.7-24. En anthropologie de la citoyenneté, voir en particulier les travaux de Catherine NEVEU : «Les enjeux d'une approche anthropologique de la citoyenneté ». Revue européenne des migrations internationales, 2004, vol. 20/4 (en ligne) ; CARREL, Marion et NEVEU, Catherine (dir.). Citoyennetés ordinaires. Pour une approche renouvelée des pratiques citoyennes. Paris: Karthala, 2014. Sur le pouvoir d'agir, voir l'approche critique de BACQUÉ, Marie-Hélène et BIEWENER, Carole. L'Empowerment, une pratique émancipatrice? Paris : La Découverte, 2013.

109. - LATOUR, Bruno. «La mondialisation fait-elle un monde habitable?». Territoire $2040, \mathrm{n}^{\circ} 2$, 2010, p. 13.

110. - CHESNEAUX, Jean. Habiter le temps. Passé, présent, futur : esquisse d'un dialogue politique. Paris : Bayard Éd., 1996.

111. - HARTOG, François. Régimes d'historicité. Présentisme et expériences du temps. Paris : Éd. du Seuil, 2003.

112. - CHESNEAUX, Jean. Op. cit., p. 73.

113. - Ibid., p. 269.

114. - HARVEY, David. Villes rebelles... Op. cit.

115. - HONNETH, Axel. La Lutte pour la reconnaissance [1992]. Paris : Éd. du Cerf, 2002 ; La Société du mépris. Vers une nouvelle théorie critique. Paris : La Découverte, 2006.

116. - FRASER, Nancy. Qu'est-ce que la justice sociale? Op. cit.

117. - BONNEUIL, Christophe. "Anthropocène... ». Art. cit., p. 29.

118. - BASCHET, Jérôme. Adieux au capitalisme. Autonomie, société du bien vivre et multiplicité des mondes. Paris : La Découverte, 2014.

119. - CASTRO, Eduardo VIVEIROS de. Métaphysiques cannibales. Op. cit.

120. - LANDIVAR, Diego et RAMILLIEN, Émilie. «La justice des "êtres possibles”. Le jour où la rivière a gagné ». Orbs. L'autre planète, 2014, n³ 3, p. 153-159; Ibid. « Reconfigurations ontologiques dans les nouvelles constitutions andines. Une analyse anthropologique». Tsantsa, 20, 2015, p. 29-40, ici p. 34-44. Voir également HARAWAY, Donna. Manifeste des espèces de compagnie. Chiens, humains et autres partenaires [2003]. Paris : Éd. de l'Éclat, 2010 (le titre français est la traduction 
très discutable du titre anglais: "The Companion Species Manifesto: Dogs, People and Significant Otherness »).

121. - ESCOBAR, Arturo. «Déplacement, développement et modernité en Colombie du Pacifique ». Revue internationale des sciences sociales, 2003/1, $\mathrm{n}^{\circ} 175, \mathrm{p} .171-182$.

122. - OGIEN, Albert et LAUGIER, Sandra. Le Principe démocratie. Enquête sur les nouvelles formes du politique. Paris : La Découverte, 2014.

123. - MOUFFE, Chantal. Agonistique. Penser politiquement le monde. Paris : Beaux-Arts de Paris Éd., 2014.

124. - GRAEBER, David. La Démocratie aux marges. Lormont: Le Bord de L'Eau, 2014. Cette exploration du lien possible entre patrimoine et anticapitalisme n'est pas sans lien avec le séminaire que j'anime depuis janvier 2016 avec Noël Barbe: Expériences de l'anticapitalisme, Ontologies et épistémologies, IIAC, EHESS, Paris.

\section{RÉSUMÉS}

La catégorie du patrimoine culturel immatériel est venue introduire l'idée, sans doute déstabilisante dans le monde culturo-académique français, de patrimoine vivant. À cela s'ajoute une tendance qui ne cesse de s'affirmer : celle de la diffusion-profusion de l'activité patrimoniale et le débordement des institutions censées organiser la relation au passé. Dans cet article, je propose de tirer les conséquences de cette double évolution : si le patrimoine est vivant, permetil de penser les grands enjeux du $\mathrm{xxI}^{\mathrm{e}}$ siècle condensés dans la notion d'Anthropocène? Si le patrimoine est entré en démocratie, participe-t-il de ces dynamiques revendicatives et alternatives parfois désignées comme citoyennes? En d'autres termes, après avoir été vu, comme un moyen de tempérer les effets destructeurs du capitalisme, le patrimoine peut-il être mobilisé dans et pour des expériences d'anticapitalisme?

The category of intangible cultural heritage has introduced the idea of living heritage, a notion that is somewhat unsettling for France's cultural and academic worlds. And this is compounded by a clear tendency which sees heritage activities multiplying and going beyond the competence and control of the institutions which are supposed to organise our relations with the past. In this article I would like to draw some conclusions from these two evolutions. If heritage is now living, will it help us face the major issues of the twenty-first century, often condensed in the notion of the Anthropocene? If heritage has been democratised, is it now part of the dynamics of alternative actions often qualified as citizens'? In other words, after having been seen as a way of tempering the destructive effects of capitalism, can heritage be mobilised in and for anticapitalist initiatives?

\section{INDEX}

Mots-clés : patrimoine, vivant, immatériel, ethnologique, citoyen, mobilisations citoyennes, Anthropocène, démocratie, anticapitalisme

Keywords : heritage, living heritage, intangible heritage, ethnology, citizen, citizens' mobilisations, Anthropocene, democracy, anti-capitalism 


\section{AUTEUR}

\section{JEAN-LOUIS TORNATORE}

Université de Bourgogne-Franche-Comté - Centre Georges-Chevrier (UMR 7366 du Cnrs) jeanlouis.tornatore@u-bourgogne.fr 